\title{
Transcutaneous Electrical Nerve Stimulation: A Review of Dental Applications
}

\author{
Vaibhav Pandita ${ }^{1}$ Vidya Ajila ${ }^{1} \quad$ G. Subhas Babu ${ }^{1}$ Shruthi Hegde ${ }^{1}$ Mohamed Faizal Asan ${ }^{1}$ \\ 1 Department of Oral Medicine and Radiology, AB Shetty Memorial \\ Institute of Dental Sciences, Nitte (Deemed to be University), \\ Mangalore, Karnataka, India

\begin{abstract}
Address for correspondence Vidya Ajila, MDS, Department of Oral Medicine and Radiology, AB Shetty Memorial Institute of Dental Sciences, Nitte (Deemed to be University), Mangalore 575018, Karnataka, India (e-mail: ajila_v@yahoo.com).
\end{abstract}

J Health Allied Sci ${ }^{\mathrm{NU}}$ 2022;12:191-195.

\begin{abstract}
Transcutaneous electrical nerve stimulation or TENS is a nonpharmacologic method that is used to alleviate pain from various acute and chronic conditions. TENS works through activation of descending inhibitory neurons of the central nervous system. In dentistry, TENS has applications for pain reduction in temporomandibular disorders and postherpetic neuralgia. It has also been studied for its role in increasing salivary secretion in subjects with xerostomia and as a local anesthetic agent in pediatric

Keywords

- transcutaneous electrical nerve stimulation

- TENS

- temporomandibular joint

- pain dentistry. Its wide applications in the orofacial region make it an important treatment modality. The purpose of the present review was to describe the general principles and its applications in dentistry along with its indications, advantages, and disadvantages. A web-based search was conducted using the keywords "transcutaneous electrical nerve stimulation," "temporomandibular joint," “TENS," "TMJ," "orofacial pain," and "Management." The manuscripts thus obtained were evaluated and used for compiling the present narrative review. The present review focuses on the mechanism of action, applications, and effectiveness of TENS in the management of diseases of the maxillofacial region.
\end{abstract}

\section{Introduction}

Transcutaneous electrical nerve stimulation or TENS is a method of peripheral nerve stimulation that is used to alleviate pain. ${ }^{1,2}$ TENS has been approved by the U.S. Food and Drug Administration. In this treatment modality, pulsed electrical current is produced in two ways; either by using direct current or batteries and is propagated along the surface of intact skin through electrodes for stimulation of superficial nerves to obtain focal pain relief. TENS is generally employed in the field of medicine in the management of acute and chronic pain. ${ }^{1,2}$ The present review focuses on the general principles of TENS therapy, mechanism of action, procedure, indications, contraindications, applications, and effectiveness of TENS in management of diseases of the maxillofacial region.

\section{Methods}

A web-based search was conducted using the keywords "transcutaneous electrical nerve stimulation," "temporomandibular joint," “TENS," “TMJ," "orofacial pain," and "Management." Databases such as "PubMed," "science direct," and "google scholar" were searched for case reports, original research, systematic reviews, and meta-analysis on TENS. Articles where full text was not available were excluded from the review. Manuscripts with information regarding the history, types of TENS therapy, mechanism of action,
DOI https://doi.org/ $10.1055 / \mathrm{s}-0041-1736275$ ISSN 2582-4287.

\footnotetext{
(C) 2021. Nitte (Deemed to be University). All rights reserved. This is an open access article published by Thieme under the terms of the Creative Commons Attribution-NonDerivative-NonCommercial-License, permitting copying and reproduction so long as the original work is given appropriate credit. Contents may not be used for commercial purposes, or adapted, remixed, transformed or built upon. (https://creativecommons.org/ licenses/by-nc-nd/4.0/)

Thieme Medical and Scientific Publishers Pvt. Ltd., A-12, 2nd Floor, Sector 2, Noida-201301 UP, India
} 
procedure, indications, contraindications, uses, and adverse effects of TENS were selected. Data extracted from the above manuscripts was evaluated and used for compiling the present narrative review.

\section{History of TENS Therapy}

The concept of pain relief by using electricity is a very old technique and not something new. During ancient times, Egyptians in 2,500 B.C used to apply electrogenic fish to parts of body that were painful. Electrostatic generators became very popular in the eighteenth century but the wide use of various pharmacological methods caused a decline in the application of this therapy by the end of nineteenth century. Melzack and Wall in 1965 concluded that the use of electrical stimulation of nerves can help in stimulation of large diameter afferent nerve fibers, thereby inhibiting central transmission of noxious stimuli. They published this in a book named "Pain Mechanisms: A New Theory." ${ }^{3}$ Clinically, it was observed that pain reduction could be achieved through stimulation of neural inhibitory pathways using electric current. TENS therapy was used initially to assess success of implants that stimulated the dorsal column. Later, it was found that TENS could be a standalone method. ${ }^{2}$

\section{Types of TENS Therapy}

There are three forms of TENS therapy: conventional TENS that uses low-intensity and high-frequency current, acupuncture-like TENS (AL-TENS) that utilizes high intensity with low frequency, and intense TENS that involves highintensity and high-frequency therapy.

\section{Conventional TENS}

The rationale behind use of conventional TENS is the selective stimulation of A-beta fibers. These are large diameter, non-noxious afferent neurons with low pain threshold. TENS stimulus causes inhibition of activity in nociceptive neurons of the central nervous system. Pain relief is attained by raising the pulse amplitude in TENS to create a nonpainful paraesthesia below the electrodes. ${ }^{4}$

\section{Acupuncture-Like TENS}

AL-TENS works through stimulation of A-delta fibers that are high-threshold peripheral afferent neurons. This causes activation of descending pain inhibitory pathways. Muscle twitching is noted during this therapy as it causes activation of various afferent nerve fibers of muscles. Electrodes placement is on acupuncture locations, over myotomes, or trigger points for pain relief. ${ }^{5}$

\section{Intense TENS}

The intense TENS is employed for the stimulation of highthreshold cutaneous A-delta fibers for the purpose of blocking propagation of painful stimulus in peripheral nerves causing activation of extrasegmental analgesic mechanisms. Intense TENS uses frequencies and high intensities that can be tolerated by patients for small time intervals. Intense
TENS behaves like a counter-irritant and is employed in minor procedures like dressing of wounds and removal of sutures. $^{2}$

Conventional TENS is used most frequently in dental practice. It can be used for the whole day with intermittent breaks to avoid skin irritation. AL-TENS uses low frequency with high amplitude and can be used for 30 minutes at a time. High-frequency and high-amplitude pulsed current is used in intense TENS therapy that is less tolerable and is not used commonly. ${ }^{6}$ The three types of TENS are summarized in -Fig. 1.

\section{TENS Procedure}

In TENS, positioning of electrodes accurately is an extremely important and time-consuming procedure. Electrodes must always be placed on intact healthy skin without altered sensation. Relevant dermatomes should be selected and sometimes units with four electrodes may be needed for widespread pain. ${ }^{2}$ There are two types of electrodes used in TENS: extraoral and intraoral. Extraoral electrodes are carbon-impregnated silicone rubber electrodes and tin plate or aluminum electrodes. Intraoral electrodes consist of cotton roll, clamp, and adhesive electrodes. ${ }^{7}$ - Fig. 2 shows a standard TENS unit.

TENS therapy is used at different frequencies, intensities, and pulse durations of stimulation. The widely followed treatment parameters include a frequency of $90 \mathrm{~Hz}$, and time duration of 30 minutes for three times a day. ${ }^{8}$ The number of treatment sessions differ widely based on the clinical requirement.

Rela and Nayyar ${ }^{9}$ used low-intensity current with an amplitude of 0 to $80 \mathrm{~Hz}$, pulse width of 1 to $11 \mu \mathrm{s}$, and pulse rate/ frequency of 0 to $11 \mathrm{~Hz}$ for TMJ pain relief. Subjects were asked to sit in upright position. Surface electrodes were placed on the sigmoid notch area and back of the neck to complete the circuit and TENS therapy was given for a duration of 45 minutes. The treatment was completed in three to four sittings including follow-up. Then the form of pain, extent of mouth opening without pain, intensity of joint, and muscle pain were measured on the visual analogue scale (VAS) and analyzed. ${ }^{9}$ Nathan and Wall ${ }^{10}$ used a frequency of $100 / \mathrm{sec}$ with pulse width of $0.1 \mathrm{msec}$ with the voltage being controlled by the subject. Twice daily to twice weekly sessions were done for 15 minutes each. Steller et al ${ }^{11}$ used 3-minute sessions of TENS thrice daily over a period of 4 weeks to manage xerostomia due to Sjogren's syndrome. In postoperative pain, frequency of 1 to $8 \mathrm{~Hz}$ for low-frequency TENS or 25 to $150 \mathrm{~Hz}$ for high-frequency TENS, with adequate intensity, that is, strong subnoxious, maximal tolerable stimulus or $>15 \mathrm{~mA}$, was associated with $30 \%$ pain relief. $^{12}$

\section{Mode of Action}

The peripheral nerves in TENS undergo antidromic activation that leads to the generation of nerve impulses. These impulses collide with and reduce afferent impulses coming from peripheral structures. The block of peripheral painful 


\begin{tabular}{|l|l|l|l|l|}
\hline \multicolumn{1}{|c|}{ Types of TENS } & \multicolumn{1}{|c|}{ Mechanism } & $\begin{array}{c}\text { Types of nerve } \\
\text { fibers stimulated }\end{array}$ & \multicolumn{1}{|c|}{ Intensity/frequency } \\
\hline Conventional TENS & $\begin{array}{l}\text { Produces segmental } \\
\text { analgesia }\end{array}$ & A-BETA fibres & $\begin{array}{l}\text { Low intensity/High frequency } \\
\text { Can be used whenever in pain }\end{array}$ & $\begin{array}{l}\text { Acute/chronic pain } \\
\text { Acute superficial pain }\end{array}$ \\
\hline Acupuncture like TENS & $\begin{array}{l}\text { Produces extra } \\
\text { Segmental analgesia }\end{array}$ & A-DELTA fibres & $\begin{array}{l}\text { High intensity/Low frequency } \\
\text { Used for approximately 15-30 minutes } \\
\text { thrice daily. }\end{array}$ & $\begin{array}{l}\text { Acute/chronic pain } \\
\text { Longstanding deep pain }\end{array}$ \\
\hline Intense TENS & $\begin{array}{l}\text { Produces extra } \\
\text { Segmental analgesia }\end{array}$ & A-DELTA fibres & High intensity/High frequency \\
Used for a few minutes & Wound dressing \\
Suture removal
\end{tabular}

Fig. 1 Comparison of different types of transcutaneous electrical nerve stimulation (TENS) therapy.

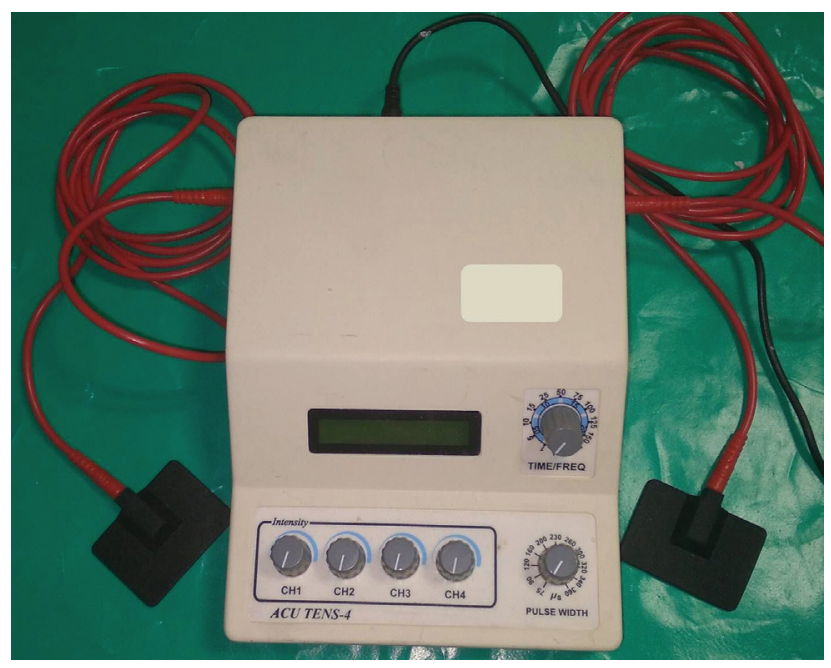

Fig. 2 Transcutaneous electrical nerve stimulation unit.

impulses is most when A-delta fibers are stimulated by TENS as it occurs in intense TENS therapy. This leads to blockage of activity of afferent nerve fibers with large diameter that are a contributing factor in pain relief (i.e., conventional TENS). The therapeutic effects of TENS therapy are produced because of various neurochemicals that include opioids, serotonin, and acetylcholine. The technique involving lowfrequency TENS therapy involves mu opioid and hydroxytryptamine receptors. The technique involving high-frequency TENS therapy involves the delta opioid receptors and decreases the spinal cord levels of aspartate and glutamate. $^{12}$

\section{Applications of TENS Therapy in Dentistry}

\section{Temporomandibular Disorders}

It has been observed that in subjects with TMD, there is increased myoelectric activity of mandibular elevator muscles when compared with normal. TENS therapy leads to decreased pain along with decreased in myoelectric activity in the muscles.

Another hypothesis is that TENS stimulates motor nerves leading to periodic muscle contractions that, in turn, cause increased perfusion and oxygen supply, decreased edema of the interstitial tissues, and increased clearance of accumulated toxins. All these events will lead to decreased pain and fatigue of muscles. ${ }^{13}$

A study was done by Shanavas et al $^{14}$ to know the effectiveness of TENS as an adjuvant therapy in temporomandibular disorders (TMD). One group was managed using medications alone, while the second group was treated with adjuvant TENS. The VAS was used to determine pain intensity. Significant pain relief was noted in the TENS group as well as controls, but when comparative analysis was performed, the use of adjunct TENS therapy was associated with more pain relief when compared with the use of drugs alone. ${ }^{15}$

Another study compared low-level laser therapy (LLLT) and TENS on improvement of restricted jaw opening in subjects with TMD. It was observed that both therapies were increased jaw opening, but TENS therapy was found to be better in causing a decreased electromyographic activity in the masseter. ${ }^{15}$

Didier et $\mathrm{al}^{16}$ studied the efficiency of the masseter and temporalis, in activity and rest, by using kinesiography to evaluate the normal mandibular rest position after TENS therapy. They employed TENS along with interocclusion devices and because of this a new position of the jaw was created. The new occlusion was associated with a significant improvement in the quality and strength of the bite, increased efficiency of the muscles, and reduced pain. ${ }^{16}$

Singh et al ${ }^{17}$ studied the usefulness of TENS versus placebo in the management of TMD. Relief of muscle pain and increased mouth opening were observed in patients receiving TENS therapy. ${ }^{17}$ Rezazadeh et al ${ }^{18}$ did a comparative study between TENS and LLLT in drug-resistant TMD. They found significant improvement in both groups. Though they reported no significant difference during treatment, TENS therapy was better in controlling pain during follow-up. ${ }^{18}$

Rodrigues et $\mathrm{al}^{19}$ performed a study using conventional TENS for muscle pain in subjects with TMD. TENS decreased pain and electromyographic activity in temporalis and masseter during maximum voluntary clenching. ${ }^{19}$ Møystad et $\mathrm{al}^{20}$ studied the effect of TENS in subjects diagnosed with rheumatic disease of the TMJ. A significant treatment effect was noted as regards with the functional and rest pain and 
tenderness of muscles and joint. ${ }^{20}$ Rela and Nayyar ${ }^{9}$ found that TENS significantly decreased pain intensity in subjects with TMJ and muscle pain. However, TENS does not bring about change in the structure or position of jaw and teeth.

\section{Dental Treatment in Pediatric Patients}

The most common observation in dental office is the negative behavior of pediatric patients because of their fear toward needles. In pedodontic subjects, TENS helps to accomplish pain control while performing various treatments including extraction and endodontic procedures. TENS therapy affects behavior of the subjects that leads to decreased anxiety because it reduces the "trypanophobia" among children. Studies show that 53 to $78 \%$ of dental professionals favor the use of TENS as compared with local anesthesia while working on pediatric patients. ${ }^{1,21}$

\section{Postherpetic Neuralgia}

In postherpetic neuralgia, large areas of myelinated nerve fibers are destroyed. Hence, the normal inhibition of pain stimulus is lost. This leads to the pain and hyperalgesia characteristic of postherpetic neuralgia. The principle behind the use of TENS is that it will compensate for the inhibitory activity by stimulating large fibers. ${ }^{10}$ Ing et $\mathrm{al}^{22}$ based on their study results found that TENS therapy was associated with pain relief in patients with recalcitrant postherpetic neuralgia. ${ }^{22}$ Kolšek $^{23}$ proposed that TENS could be used as an adjunct or as a replacement for antivirals in management of herpes zoster. They further stated that TENS appeared to be more effective in postherpetic neuralgia prevention. ${ }^{23}$ The beneficial effect of TENS could be due to the endogenous opioid release as well as segmental inhibition in the dorsal horn of the spinal cord. ${ }^{24}$

\section{Xerostomia}

Regulation of salivary secretion is under the control of autonomic nervous system that includes sympathetic as well as parasympathetic efferent fibers. TENS directly causes stimulation of the auriculotemporal nerve that supplies the parotid gland. ${ }^{11}$ TENS can cause endorphin release in opiate receptors thereby increasing salivary flow. ${ }^{25}$ Sympathetic stimulation produces scanty and viscous saliva for which higher frequencies and longer duration of pulse are needed. Contrary to this, parasympathetic stimulation at lower frequencies generates higher amounts of watery saliva. This higher quantity of serous saliva is useful in the management of xerostomia. ${ }^{11}$

Sivaramakrishnan and Sridharan, ${ }^{26}$ in their systematic review, found no significant rise in the flow of saliva after TENS therapy. However, they observed that it could be used as a salivary aid to obtain symptomatic improvement. ${ }^{26}$

\section{Other Applications}

TENS has multiple applications in both acute and chronic pain. Examples include trauma, angina, cancer-related pain, and any form of musculoskeletal pain. ${ }^{2}$

\section{Advantages of TENS Therapy}

The chief advantage of employing TENS is that it is noninvasive in contrast to drugs, which have their systemic adverse effects. ${ }^{26}$ Decrease in pain and tenderness is relatively faster with TENS. It is associated with improved joint function. TENS can be used in anesthesia for trypanophobic subjects. In contrast to local anesthesia, postoperative anesthesia is experienced by the subject once the TENS unit is switched off.

Subjects can administer TENS themselves and modify dosages according to their need. This has increased acceptance of this modality.

\section{Disadvantages of TENS Therapy}

TENS cannot be used in patients with cardiac pacemakers, pregnancy, and bleeding disorders. TENS therapy is ineffective in temporomandibular pain caused due to recent trauma, dental surgery, and metabolic diseases. ${ }^{25}$ In few cases, mild skin irritation has been noted below the electrodes. $^{27}$

\section{Precautions}

Certain precautions need to be taken before and during the use of TENS. The unit should be turned off when placing electrodes over intact skin. Electrodes should not be placed over the anterior neck as it can precipitate laryngeal spasm or a hypotensive response. Electrodes should not be placed over the anterior and posterior chest as it can stimulate intercostal muscles causing impaired pulmonary function. Other areas to be avoided include sites of recent hemorrhage, open wounds, ischemic tissue, and thrombosis. ${ }^{27}$ When TENS is used for pain relief in malignancy, it should always be under the supervision of a specialist. In areas with altered pain sensation, TENS must be used with caution to avoid skin damage and increased pain. ${ }^{27}$

\section{Conclusion}

TENS therapy has a vast potential and scope in medical and dental specialities. It not only helps in pain relief but also helps in managing various conditions affecting the oral and maxillofacial region. TENS cannot be a replacement to local anesthesia, but it can be used like an adjunct to it in dental office. Based on the above review, we can conclude that TENS is an easy, cost-effective modality for pain management in the head and neck region with minimal side effects and the potential for self-administration. More research needs to be performed to explore the multiple applications and scope of TENS in dentistry.

Conflict of Interest

None declared. 


\section{References}

1 Dhindsa A, Pandit IK, Srivastava N, Gugnani N. Comparative evaluation of the effectiveness of electronic dental anesthesia with $2 \%$ lignocaine in various minor pediatric dental procedures: a clinical study. Contemp Clin Dent 2011;2(01):27-30

2 Johnson M. Transcutaneous electrical nerve stimulation: mechanisms, clinical application and evidence. Rev Pain 2007;1(01): 7-11

3 Melzack R, Wall PD. Pain mechanisms: a new theory. Science 1965;150(3699)971-979

4 Charlton J. Core Curriculum for Professional Education in Pain. 3rd edition Seattle: IASP Press; 2005:93-96

5 Johnson MI. Acupuncture-like transcutaneous electrical nerve stimulation (AL-TENS) in the management of pain. Phys Ther Rev 1998;3:73-93

6 Banerjee G, Jhonson MI. Transcutaneous electrical nerve stimulation (TENS). A potential intervention for pain management in India? Indian J Pain 2013;27:132-141

7 Kasat V, Gupta A, Ladda R, Kathariya M, Saluja H, Farooqui AA. Transcutaneous electric nerve stimulation (TENS) in dentistry- a review. J Clin Exp Dent 2014;6(05):e562-e568

8 Linde C, Isacsson G, Jonsson BG. Outcome of 6-week treatment with transcutaneous electric nerve stimulation compared with splint on symptomatic temporomandibular joint disk displacement without reduction. Acta Odontol Scand 1995;53(02):92-98

9 Rela R, Nayyar AS. Transcutaneous electric nerve stimulation (TENS) as pain management in TMD patients: a cross sectional study. Int J Appl Dent Sci 2018;4:298-301

10 Nathan PW, Wall PD. Treatment of post-herpetic neuralgia by prolonged electric stimulation. BMJ 1974;3(5932):645-647

11 Steller M, Chou L, Daniels TE. Electrical stimulation of salivary flow in patients with Sjögren's syndrome. J Dent Res 1988;67(10): 1334-1337

12 Vance CG, Dailey DL, Rakel BA, Sluka KA. Using TENS for pain control: the state of the evidence. Pain Manag 2014;4(03): 197-209

13 Grossmann E, Tambara JS, Grossmann TK, Siqueira JTT. Transcutaneous electrical nerve stimulation for temporomandibular joint dysfunction. Rev Dor 2012;13:271-276

14 Shanavas M, Chatra L, Shenai P, et al. Transcutaneous electrical nerve stimulation therapy: an adjuvant pain controlling modality in TMD patients - a clinical study. Dent Res J (Isfahan) 2014;11 (06):676-679
15 Núñez SC, Garcez AS, Suzuki SS, Ribeiro MS. Management of mouth opening in patients with temporomandibular disorders through low-level laser therapy and transcutaneous electrical neural stimulation. Photomed Laser Surg 2006;24(01):45-49

16 Didier H, Marchetti C, Borromeo G, Tullo V, Bussone G, Santoro F. Persistent idiopathic facial pain: multidisciplinary approach and assumption of comorbidity. Neurol Sci 2010;31(Suppl 1): S189-S195

17 Singh H, Sunil MK, Kumar R, Singla N, Dua N, Garud SR. Evaluation of TENS therapy and Placebo drug therapy in the management of TMJ pain disorders: a comparative study. J Indian Acad Oral Med Radiol 2014;26:139-144

18 Rezazadeh F, Hajian K, Shahidi S, Piroozi S. Comparison of the effects of transcutaneous electrical nerve stimulation and lowlevel laser therapy on drug-resistant temporomandibular disorders. J Dent (Shiraz) 2017;18(03):187-192

19 Rodrigues D, Siriani AO, Bérzin F. Effect of conventional TENS on pain and electromyographic activity of masticatory muscles in TMD patients. Braz Oral Res 2004;18(04):290-295

20 Møystad A, Krogstad BS, Larheim TA. Transcutaneous nerve stimulation in a group of patients with rheumatic disease involving the temporomandibular joint. J Prosthet Dent 1990;64(05): 596-600

21 Baghdadi ZD. Evaluation of electronic dental anesthesia in children. Oral Surg Oral Med Oral Pathol Oral Radiol Endod 1999;88 (04):418-423

22 Ing MR, Hellreich PD, Johnson DW, Chen JJ. Transcutaneous electrical nerve stimulation for chronic post-herpetic neuralgia. Int J Dermatol 2015;54(04):476-480

23 Kolšek M. TENS - an alternative to antiviral drugs for acute herpes zoster treatment and postherpetic neuralgia prevention. Swiss Med Wkly 2012;141:w13229

24 Christo PJ, Hobelmann G, Maine DN. Post-herpetic neuralgia in older adults: evidence-based approaches to clinical management. Drugs Aging 2007;24(01):1-19

25 Yap AU, Ho HC. Electronic and local anesthesia: a clinical comparison for operative procedures. Quintessence Int 1996;27(08): 549-553

26 Sivaramakrishnan G, Sridharan K. Electrical nerve stimulation for xerostomia: a meta-analysis of randomised controlled trials. J Tradit Complement Med 2017;7(04):409-413

27 Jones I, Johnson MI. Transcutaneous electrical nerve stimulation. Contin Educ Anaesth Crit Care Pain 2009;9:130-135 